\title{
Photoinduced Electron Transfer and Solvation in Iodide-doped Acetonitrile Clusters ${ }^{\dagger}$
}

\author{
Oli T. Ehrler, Graham B. Griffin, Ryan M. Young, and Daniel M. Neumark* \\ Department of Chemistry, University of California, Berkeley, California 94720 and Chemical Sciences \\ Division, Lawrence Berkeley National Laboratory, Berkeley, California 94720
}

Received: July 31, 2008; Revised Manuscript Received: September 25, 2008

\begin{abstract}
We have used ultrafast time-resolved photoelectron imaging to measure charge transfer dynamics in iodidedoped acetonitrile clusters $\mathrm{I}^{-}\left(\mathrm{CH}_{3} \mathrm{CN}\right)_{n}$ with $n=5-10$. Strong modulations of vertical detachment energies were observed following charge transfer from the halide, allowing interpretation of the ongoing dynamics. We observe a sharp drop in the vertical detachment energy (VDE) within 300-400 fs, followed by a biexponential increase that is complete by $\sim 10 \mathrm{ps}$. Comparison to theory suggests that the iodide is internally solvated and that photodetachment results in formation of a diffuse electron cloud in a confined cavity. We interpret the initial drop in VDE as a combination of expansion of the cavity and localization of the excess electron on one or two solvent molecules. The subsequent increase in VDE is attributed to a combination of the I atom leaving the cavity and rearrangement of the acetonitrile molecules to solvate the electron. The $n$ =5-8 clusters then show a drop in VDE of around $50 \mathrm{meV}$ on a much longer time scale. The long-time VDEs are consistent with those of $\left(\mathrm{CH}_{3} \mathrm{CN}\right)_{n}{ }^{-}$clusters with internally solvated electrons. Although the excitedstate created by the pump pulse decays by emission of a slow electron, no such decay is seen by 200 ps.
\end{abstract}

\section{Introduction}

Solvated electrons and, in particular, hydrated electrons play a decisive role in topics ranging from formation of reactive species in marine aerosols ${ }^{1,2}$ to the radiative damage of DNA. ${ }^{3,4}$ The development of ultrafast lasers has led to new insights into the formation and reaction dynamics of solvated electrons. ${ }^{5}$ Although most of these studies have been carried out in water, experiments have been carried out in other dipolar solvents including alcohols, ${ }^{6-8}$ ethers, ${ }^{9-11}$ and other polar molecules. ${ }^{12-14}$ In several of these experiments, ${ }^{6,8,11,15,16}$ solvated electrons were generated by photodetachment of dissolved negative ions, most commonly halide ions, via excitation of charge-transfer-tosolvent (CTTS) bands in which the electron is ejected from the halide into the surrounding solvent. Electron solvation dynamics are detected by changes in the transient absorption spectrum. The time evolution of this signal directly probes the dynamics of electron solvation. In this paper, we perform the gas phase analog of the CTTS experiment by performing time-resolved experiments on clusters in which an iodide anion is surrounded by a known number of acetonitrile molecules. These experiments are motivated by our past time-resolved experiments on $\mathrm{I}^{-}\left(\mathrm{H}_{2} \mathrm{O}\right)_{n}$ clusters ${ }^{17-19}$ the solvent-binding motifs of both the iodide and ejected electron are expected to be very different in $\mathrm{I}^{-}\left(\mathrm{CH}_{3} \mathrm{CN}\right)_{n}$ clusters.

The iodide anion in liquid acetonitrile has two CTTS bands centered at 245 and $210 \mathrm{~nm} \cdot{ }^{20}$ Photodetachment of iodide in bulk acetonitrile has been studied by Xia et $\mathrm{al}^{20}$ in a fs pump-probe experiment by pumping the higher wavelength band and monitoring the resulting transient absorption spectrum. Two transient features were found centered at around 500 and $1500 \mathrm{~nm}$, and these were attributed to two solvation motifs of the ejected electron; the $500 \mathrm{~nm}$ band was assigned to formation of a valence anion, in which the electron is localized on one or

\footnotetext{
$\dagger$ Part of the special section "Aqueous Solutions and Their Interfaces".

* Author to whom correspondence should be addressed. E-mail: dneumark@berkeley.edu.
}

two solvent molecules, and the $1500 \mathrm{~nm}$ band was ascribed to a solvated electron in a solvent cavity, more akin to the hydrated electron. Shkrob and Sauer ${ }^{21}$ independently found similar results by pulse radiolysis-transient absorption and time-resolved photoconductivity.

Evidence for two different electron solvation motifs in acetonitrile also comes from gas phase photoelectron (PE) spectroscopy measurements by Mitsui et al. ${ }^{22}$ on bare $\left(\mathrm{CH}_{3} \mathrm{CN}\right)_{n}{ }^{-}$anions, $n=10-100$. Two isomers were observed: isomer I, with vertical detachment energies (VDEs) ranging from 0.4 to $1.0 \mathrm{eV}$; and isomer II, with considerably higher binding energies between 2.5 and $3.0 \mathrm{eV}$. Isomer I dominated the PE spectra for $n=10-12$, whereas the signal from isomer II was somewhat larger for $n=13$ and was larger by 2 orders of magnitude for $n \geq 30$. Assignments were made in reference to the condensed phase studies. Structure II was attributed to a valence-bound isomer with the excess charge being localized on an acetonitrile dimer inside the cluster and distributed over the $\pi^{*}$ orbitals of two antiparallel oriented solvent molecules, giving rise to the large binding energy. In contrast, the more weakly bound isomer I was assigned to an electron within a solvent cavity. Density functional computations of acetonitrile cluster anions up to $n=10$ supported the existence of both structural motifs and the assignments based on the VDEs. ${ }^{23,24}$

Iodide-acetonitrile clusters have been investigated by Dessent et al., ${ }^{25}$ who measured the electronic spectra of $\mathrm{I}^{-}\left(\mathrm{CH}_{3} \mathrm{CN}\right)_{n}(n$ $=1,2$ ) and by Markovich et al., ${ }^{26}$ who measured anion PE spectra for clusters as large as $n=55$. Trends in the VDEs from the PE spectra and accompanying molecular dynamics simulations indicated that the iodide was internally solvated, with the first solvation shell closing at $n=12$. Subsequent Monte Carlo simulations by Peslherbe ${ }^{27,28}$ on large clusters (up to $n=36$ ) also found internally solvated structures to be the most stable, while electronic structure calculations by Peslherbe ${ }^{28}$ and Takayanagi ${ }^{29}$ located the iodide at the center of the $n=2$ and 3 clusters. 
The experimental and theoretical results on both $\mathrm{I}^{-}\left(\mathrm{CH}_{3} \mathrm{CN}\right)_{n}$ and $\left(\mathrm{CH}_{3} \mathrm{CN}\right)_{n}{ }^{-}$are of considerable interest in comparison to the analogous water-containing clusters. There is now a consensus that the iodide anion sits at the surface of $\mathrm{I}^{-}\left(\mathrm{H}_{2} \mathrm{O}\right)_{n}$ clusters, at least for clusters as large as $n=32 .{ }^{30,31}$ The situation for water cluster anions, $\left(\mathrm{H}_{2} \mathrm{O}\right)_{n}{ }^{-}$, is more complex. ${ }^{32} \mathrm{PE}$ spectra in our group showed evidence for at least two structural isomers, ${ }^{33-35}$ I and II over a wide size range $(n \leq 200)$, with considerably lower VDEs seen for isomer II clusters. On the basis of previous theoretical work, ${ }^{36}$ isomer II clusters were assigned to surface-bound electrons, whereas isomer I was assigned to internally solvated electrons, in agreement with the earlier assignment by Bowen and co-workers ${ }^{37}$ who originally measured PE spectra for isomer I clusters. However, this assignment of isomer I clusters has become somewhat controversial in light of more recent theoretical and experimental results, ${ }^{38-41}$ and there is now reasonably strong evidence that the excess electron resides at the surface for isomer I clusters as large as $n=25 .{ }^{42}$

The iodide anion in aqueous solution exhibits a CTTS band around $225 \mathrm{~nm} .{ }^{43}$ The analog of this transition in iodide-water clusters, which occurs at somewhat lower energies, was identified by Serxner et al. ${ }^{44}$ Previous work in our group made use of CTTS absorption to directly observe photoinduced formation of a free excess charge in doped water clusters $\mathrm{I}^{-}\left(\mathrm{H}_{2} \mathrm{O}\right)_{n}$ as large as $n=28$ by means of time-resolved photoelectron spectroscopy. ${ }^{17-19}$ In these experiments, the cluster CTTS transition was excited by a femtosecond pump pulse at either 266 or $240 \mathrm{~nm}$; the evolving system was photodetached by a fs probe pulse at various delay times and the resulting PE spectrum was measured. These experiments showed a marked increase in the VDE, as large as $0.7 \mathrm{eV}$ for the $n=28$ cluster, on a time scale of $\sim 1 \mathrm{ps}$. This increase was attributed to rearrangement of the water molecules to solvate the electron injected into the water network by the CTTS excitation. A slower destabilization of $\sim 50 \mathrm{meV}$ over the following tens of picoseconds was attributed to the neutral iodine atom leaving the cluster. Excited clusters were metastable and decayed by emission of slow electrons with lifetimes between 8 ps and 3 ns. ${ }^{19}$ VDEs measured shortly after and long after the pump pulse were quite close to those for isomers II and I of bare water cluster anions, respectively, suggesting that CTTS excitation created an electron binding motif similar to that of isomer II, that is, a diffuse surface state, that spontaneously isomerized to the more tightly bound isomer I motif. However, it would appear that these dynamics occur mostly at the surface of the cluster, given that the iodide is initially at the surface and that, in this size range, the excess electron in isomer I clusters is at or near the surface rather than inside a solvent cavity.

In this paper, we study ultrafast dynamics following excitation of the CTTS transition in molecular clusters $\mathrm{I}^{-}\left(\mathrm{CH}_{3} \mathrm{CN}\right)_{n}$ with $5 \leq n \leq 10$ by time-resolved photoelectron imaging. Here, both the initial and final conditions are expected to be very different from $\mathrm{I}^{-}\left(\mathrm{H}_{2} \mathrm{O}\right)_{n}$ clusters, because both the $\mathrm{I}^{-}$and, at long times, the excess electron should be internally solvated. Our experiments are interpreted through comparison with the experimental studies discussed above and theoretical work on CTTS excitation in $\mathrm{I}^{-}\left(\mathrm{CH}_{3} \mathrm{CN}\right)_{n}$ clusters and the subsequent dynamics. ${ }^{29,45}$ This comparison suggests that photodetachment results in the formation of a diffuse electron cloud in a confined cavity. A rapid initial drop in VDE, occurring within 300-400 fs, is interpreted as a combination of expansion of the cavity and localization of the excess electron on one or two solvent molecules. The subsequent increase in VDE occurring over the next $10 \mathrm{ps}$ is attributed to a combination of the I atom leaving the cavity and rearrangement of the acetonitrile molecules to solvate the electron. The long-time VDEs are consistent with those of $\left(\mathrm{CH}_{3} \mathrm{CN}\right)_{n}{ }^{-}$clusters with internally solvated excess electrons.

\section{Experimental}

Our experimental setup has been described previously, ${ }^{46,47}$ and only a brief summary is given here. Cluster ions were created by supersonic expansion of 40-50 psi of Argon gas, which was bubbled through liquid acetonitrile at $0{ }^{\circ} \mathrm{C}$ and then passed over a methyl iodide sample at room temperature, through a piezoelectric pulsed valve operated at $500 \mathrm{~Hz}$. The molecular beam interacted with electrons emitted from a pulsed ring ionizer operated at $250-500 \mathrm{~V}$, forming iodide by dissociative secondary electron attachment to the $\mathrm{CH}_{3} \mathrm{I}$. The anions were extracted in a perpendicular Wiley/McLaren timeof-flight setup and separated according to their mass-to-charge ratio, and the species of interest was isolated with a pulsed high voltage electrode. In the center of the velocity map lens, the mass-selected ions were then irradiated with pump and probe laser beams, which were transmitted into the vacuum chamber through a thin fused silica window. The resulting electron cloud was projected onto a $70 \mathrm{~mm}$ chevron type dual microchannel plate detector coupled to a phosphor screen. The resulting 2-dimensional images were captured with a charge-coupled device camera and integrated on a personal computer.

The output of a commercial regeneratively amplified Ti: Sapphire femtosecond laser system (Clark-MXR, NJA-5 and CPA-1000) operating at $795 \mathrm{~nm}$ was used as a probe, and various frequency conversion schemes were used to generate pump laser pulses suitable to excite the CTTS transition in molecular clusters of differing sizes. Clusters comprising $n=$ 5-8 acetonitrile molecules were pumped by the third harmonic of the laser (at a wavelength of $265 \mathrm{~nm}, 30 \mu \mathrm{J} /$ pulse), while clusters with $n=9$ and 10 were pumped by photons created by parametric conversion of the fundamental (TOPAS, Light Conversion Ltd.), followed by sum-frequency mixing of the signal with remaining fundamental and subsequent second harmonic generation, resulting in a wavelength of $245 \mathrm{~nm}$ (with $5 \mu \mathrm{J} /$ pulse). Typical pulse durations from the Clark system were less than $100 \mathrm{fs}$, and frequency conversion only led to minor pulse stretching, resulting in a final temporal resolution of 120-200 fs, as measured by up-conversion in a nonlinear crystal. Pulse intensities were carefully chosen to allow for sufficient amounts of $\left[1+1^{\prime}\right]$ signal, while suppressing degenerate two-photon signals.

One photoelectron was typically collected for every $1-10$ laser shots, depending on cluster size, and PE images were integrated over 30 000-600 000 shots at each delay. We applied four-way symmetrization to the images to account for detector inhomogeneities, and reconstructed the 3-dimensional electron emission distribution by expansion in a set of basis functions (BASEX). ${ }^{48}$ PE spectra were obtained by angular integration of the resulting velocity distributions, and spectra were calibrated daily to the atomic detachment transitions of $\mathrm{I}^{-}$. The energy resolution of our setup was around $5 \%$.

\section{Results}

Figures 1 and 2 show time-resolved photoelectron spectra for clusters $\mathrm{I}^{-}\left(\mathrm{CH}_{3} \mathrm{CN}\right)_{n}, n=5$ and 10, respectively. Photoelectron yields are plotted as false color images as a function of both electron kinetic energy (eKE) and pump/probe delay $t$. Although eKE is shown on a linear scale between zero and 2.0 $\mathrm{eV}$, a nonlinear scale was chosen for the delay time axis to allow 


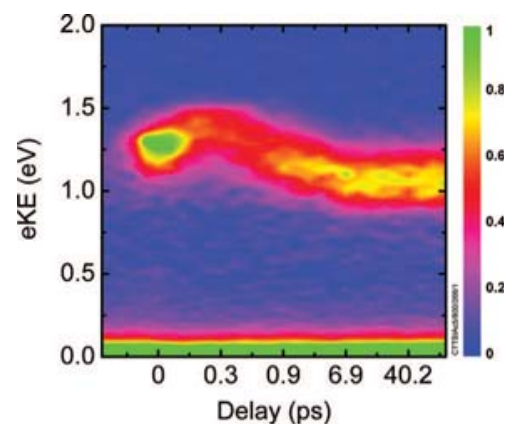

Figure 1. False color map of time-resolved photoelectron spectra of $\mathrm{I}^{-}\left(\mathrm{CH}_{3} \mathrm{CN}\right)_{5}$ taken at $h v_{\text {pump }}=4.68 \mathrm{eV}$ and $h v_{\text {probe }}=1.56 \mathrm{eV}$. Electron yield as a function of kinetic energy is plotted against pump/probe delay along the horizontal axis.

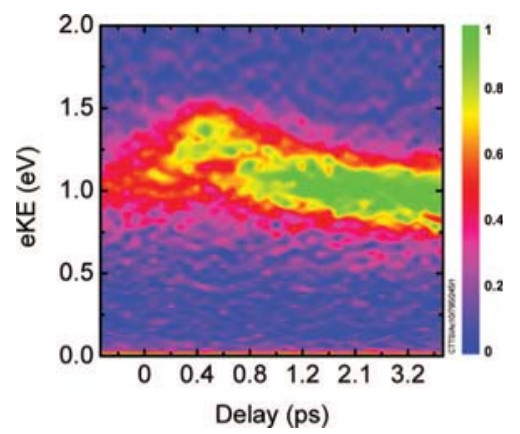

Figure 2. False color map of time-resolved photoelectron spectra of $\mathrm{I}^{-}\left(\mathrm{CH}_{3} \mathrm{CN}\right)_{10}$ taken at $h v_{\text {pump }}=5.06 \mathrm{eV}$ and $h v_{\text {probe }}=1.56 \mathrm{eV}$. Electron yield as a function of kinetic energy is plotted against pump/probe delay along the horizontal axis.

for better discrimination of early dynamics. The pump energies for $\mathrm{I}^{-}\left(\mathrm{CH}_{3} \mathrm{CN}\right)_{5}$ in Figure 1 and $\mathrm{I}^{-}\left(\mathrm{CH}_{3} \mathrm{CN}\right)_{10}$ (Figure 2) were 4.68 and $5.06 \mathrm{eV}$, respectively, and the same probe energy, 1.56 $\mathrm{eV}$, was used in all spectra. The higher pump energy was needed for the $n=9$ and 10 clusters since they did not absorb enough light at $4.68 \mathrm{eV}$ to produce sufficient signal.

Just after excitation with the pump pulse (i.e., for $t \geq 0$ ), photoelectron spectra show a $\left[1+1^{\prime}\right]$ detachment feature around $\mathrm{eKE}=1.25 \mathrm{eV}$, resulting from both one pump and probe photon. Over the following 300-400 fs, this excited-state feature shifts to higher kinetic energies and reaches a maximum value at $\mathrm{eKE}=1.4 \mathrm{eV}$. The eKE then decreases over the next few picoseconds, reaching a minimum smaller than the initial eKE. For the $n=5$ cluster in Figure 1, the eKE distribution shifts to slightly higher values over several hundred picoseconds; no such shift is seen for $\mathrm{I}^{-}\left(\mathrm{CH}_{3} \mathrm{CN}\right)_{10}$. The PE yield for excited-state detachment of the $\mathrm{I}^{-}\left(\mathrm{CH}_{3} \mathrm{CN}\right)_{5}$ cluster is highest just after absorption of the pump photon, then drops and remains essentially constant throughout the examined time range. In contrast, the electron yield for excited-state detachment of the $n=10$ cluster increases rapidly after excitation and then remains roughly constant throughout the measurement. These intensity trends are more apparent in the plots shown in the following section.

Photoelectron spectra show a strong signal around $\mathrm{eKE} \approx 0$ $\mathrm{eV}$, at the bottom of the plots, at all times. It is present even when the probe laser pulse is absent and follows a single photon power dependence of the UV excitation pulse. Color scales in Figures 1 and 2 are chosen to optimally visualize the excitedstate feature. Therefore, they obscure the fact that peak values of the slow electron signal exceed the maximum excited-state intensity by at least an order of magnitude. A sharp drop of slow electron emission is observable after the probe pulse fires,

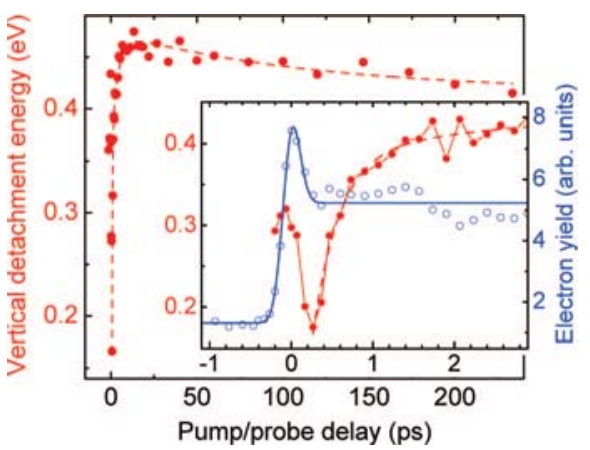

Figure 3. Evolution of vertical detachment energies (filled red circles and dashed lines) and excited-state detachment yield (blue empty circles and solid line) of $\mathrm{I}^{-}\left(\mathrm{CH}_{3} \mathrm{CN}\right)_{5}$ measured at $h v_{\text {pump }}=4.68 \mathrm{eV}$ and $h v_{\text {probe }}$ $=1.56 \mathrm{eV}$. Dots are experimental values, and curves are least-squares fits, as described in the text.

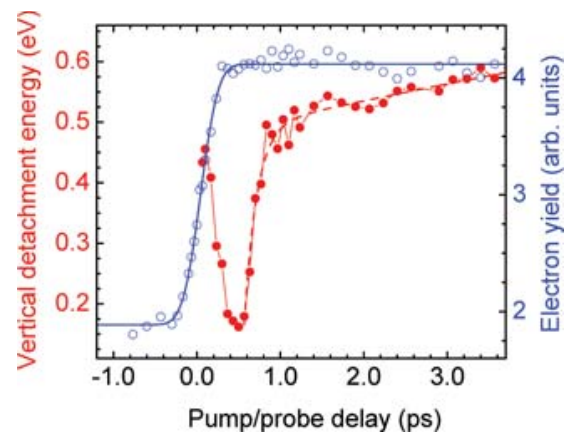

Figure 4. Evolution of vertical detachment energies (filled red circles and dashed line) and excited-state detachment yield (blue empty circles and solid line) of $\mathrm{I}^{-}\left(\mathrm{CH}_{3} \mathrm{CN}\right)_{10}$ measured at $h v_{\text {pump }}=5.06 \mathrm{eV}$ and $h v_{\text {probe }}$ $=1.56 \mathrm{eV}$. Dots are experimental values, and curves are least-squares fits, as described in the text.

but quantitative analysis is hindered by saturation of the detector at very small eKE values.

\section{Analysis}

In this section, the two-dimensional (2D) plots in Figures 1 and 2 are analyzed in more detail by determining how the overall photoelectron intensities and photoelectron kinetic energy distributions vary with pump-probe delay. Results at longer pump-probe delays than shown in the $2 \mathrm{D}$ plots are also presented.

The integrated photoelectron yield from excited-state detachment is plotted as a function of delay time $t$ for clusters with $n$ $=5$ and 10 in Figures 3 and 4, respectively (blue empty circles). For $\mathrm{I}^{-}\left(\mathrm{CH}_{3} \mathrm{CN}\right)_{5}$, the electron yield peaks at time zero (inset of Figure 3 ) then falls off and remains practically constant over the following hundreds of picoseconds. In contrast, for $n=10$ clusters the excited-state intensity reaches its maximum after excitation and stays constant at this value (Figure 4). Both contributions are convolved with the temporal resolution of our experiment and were fitted according to eq 1 ,

$$
I(t)=A[1+\operatorname{erf}(t / \varepsilon)]+B \exp \left[-(t / \varepsilon)^{2}\right]+C
$$

with $\varepsilon=150$ fs reflecting the cross-correlation between pump and probe laser pulses. Although a peak in the detachment yield around time zero was observed for clusters with $n=5$ and 6 , its relative amplitude was lower for the larger cluster. Timedependences for the larger clusters $(n=7-10)$ follow only the error function $(B=0)$. Least-squares fits to eq 1 for the $n=5$ 


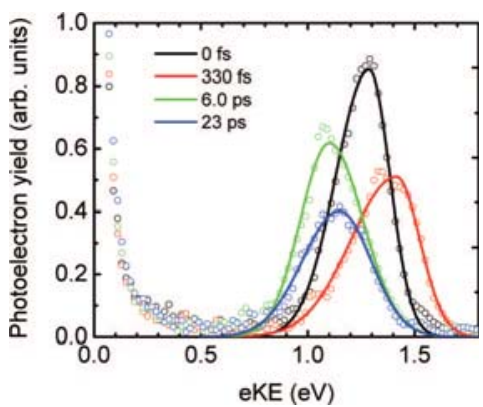

Figure 5. Photoelectron spectra of $\mathrm{I}^{-}\left(\mathrm{CH}_{3} \mathrm{CN}\right)_{5}$ taken after excitation at $h v_{\text {pump }}=4.68 \mathrm{eV}$ and with detachment photon energy $h v_{\text {probe }}=1.56$ $\mathrm{eV}$. Electron yield is plotted as a function of kinetic energy for various pump/probe delays. Dots reflect experimental data, and solid lines are least-squares fits of an asymmetric Gaussian distribution.

and 10 clusters are shown as solid blue lines in Figures 3 and 4 , respectively.

We can also analyze the time dependence of energetics following excitation of the charge-transfer state. Although Figures 1 and 2 diagrammatically reflect the change in electron binding energies over the course of the experiment, we have determined time-dependent vertical values by a least-squares fit routine to quantitatively describe the dynamics. To account for the asymmetrically broadened shape of the excited-state detachment signals, we used a modified Gaussian function with different time-dependent widths $\varepsilon_{1}$ and $\varepsilon_{2}$ on either side of the maximum at $E_{0}(t)$ to analyze our data.

$$
\begin{aligned}
& I(\mathrm{eKE}, t)= \\
& \begin{cases}I_{\mathrm{CTTS}}(t) \times \exp \left[-\left(\frac{\mathrm{eKE}-E_{0}(t)}{\varepsilon_{1}(t)}\right)^{2}\right] & \text { if eKE } \leq E_{0} \\
I_{\mathrm{CTTS}}(t) \times \exp \left[-\left(\frac{\mathrm{eKE}-E_{0}(t)}{\varepsilon_{2}(t)}\right)^{2}\right] & \text { if eKE }>E_{0}\end{cases}
\end{aligned}
$$

This modified Gaussian distribution fits the excited-state signal at all delays. Representative spectra of $\mathrm{I}^{-}\left(\mathrm{CH}_{3} \mathrm{CN}\right)_{5}$ at various times, along with the optimized fit functions are shown in Figure 5.

Kinetic energies $E_{0}$ were converted to vertical detachment energies according to $\operatorname{VDE}(t)=h v_{\text {probe }}-E_{0}$, using the probe photon energy of $1.56 \mathrm{eV}$. Values for the excited-state feature in clusters $\mathrm{I}^{-}\left(\mathrm{CH}_{3} \mathrm{CN}\right)_{5}$ and $\mathrm{I}^{-}\left(\mathrm{CH}_{3} \mathrm{CN}\right)_{10}$ are plotted as filled red circles in Figures 3 and 4, respectively, along with the detachment yield.

The VDE of the CTTS feature in $\mathrm{I}^{-}\left(\mathrm{CH}_{3} \mathrm{CN}\right)_{5}$ directly after absorption is $\operatorname{VDE}(0)=0.30 \mathrm{eV}$, which decreases over the following $330 \mathrm{fs}$ to reach its minimum $\mathrm{VDE}_{\min }=0.18 \mathrm{eV}$ at delay $t_{\min }$ (see inset in Figure 3 ). The subsequent rise leads to the maximum detachment energy $\mathrm{VDE}_{\max }=0.48 \mathrm{eV}$ at $t_{\max }$ and is finally followed by a slow decrease to $\operatorname{VDE}(\infty)=0.42$ $\mathrm{eV}$, which is reached within less than 200 ps (Figure 3). The same VDE is also found in a pump-probe spectrum taken at the longer delay $t=400 \mathrm{ps}$ (not shown). Similarly, a final slow decrease was seen for VDEs in clusters with $n=6-8$. Detachment energies in the larger cluster $\mathrm{I}^{-}\left(\mathrm{CH}_{3} \mathrm{CN}\right)_{10}$ show an initial value $\operatorname{VDE}(0)=0.43 \mathrm{eV}$ (Figure 4), which steeply decreases to $\mathrm{VDE}_{\min }=0.17 \mathrm{eV}$ at $t_{\min }=500$ fs to subsequently increase back to $\mathrm{VDE}_{\max }=0.58 \mathrm{eV}$. No further change is seen for at least $\sim 400 \mathrm{ps}$. The same long-time behavior was found for clusters with $n=9$.

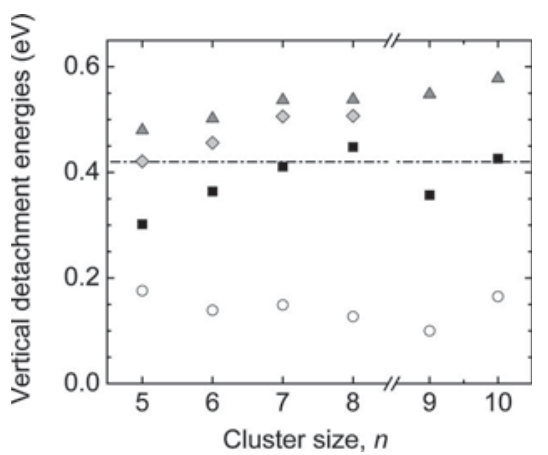

Figure 6. Vertical detachment energies in the course of relaxation following excitation of the CTTS transition in $\mathrm{I}^{-}\left(\mathrm{CH}_{3} \mathrm{CN}\right)_{n}$. Black squares are initial detachment energies $\operatorname{VDE}(0)$, and dots and dark gray triangles are $\mathrm{VDE}_{\min }$ and $\mathrm{VDE}_{\max }$, respectively. The light-gray diamonds reflect detachment energies $\operatorname{VDE}(\infty)$ at large delay times, where appropriate. The dashed-dotted line corresponds to the VDE of isomer I of bare acetonitrile cluster anions comprising 10 molecules (as taken from ref 22).

The temporal evolution of $\operatorname{VDE}(t)$ after reaching the minimum $\mathrm{VDE}_{\min }$ at $t_{\min }$ could be quantitatively modeled by a multiexponential decay according to eq 3 .

$$
\operatorname{VDE}(t)=\sum_{i} E_{i} \exp \left(-\left(t-t_{\min }\right) / \tau_{i}\right)+\operatorname{VDE}(\infty)
$$

Two exponential terms are necessary to describe the increase toward $\mathrm{VDE}_{\max }$, and lifetimes of $\tau_{1}=250 \pm 100 \mathrm{fs}$ and $\tau_{2}=$ $2.5 \pm 1.0 \mathrm{ps}$ were found throughout the whole cluster size range. A third term with negative prefactor, $E_{3}$, was necessary to describe the final decrease of VDEs toward $\operatorname{VDE}(\infty)$ in clusters with $n=5-8$. Lifetimes on the order of $\tau_{3} \approx 100-150 \mathrm{ps}$ could be determined in these cases, but errors are large as the lifetimes approach the time window of our experiments. The resulting maximum in $\operatorname{VDE}(t)$ was used to determine the values of $t_{\max }$. Least-squares fits to eq 3 for clusters with $n=5$ and 10 are shown in Figures 3 and 4, respectively.

A compilation of characteristic detachment energies $\operatorname{VDE}(0)$, $\mathrm{VDE}_{\text {min }}$, and $\mathrm{VDE}_{\text {max }}$ (and $\operatorname{VDE}(\infty)$ for clusters where it is less than $\mathrm{VDE}_{\max }$ ) for all clusters measured are shown in Figure 6. Table 1 shows numerical values, along with energy shifts $\Delta E_{1}$ $=\mathrm{VDE}_{\min }-\mathrm{VDE}(0), \Delta E_{2}=\mathrm{VDE}_{\max }-\mathrm{VDE}_{\min }$ and $\Delta E_{3}=$ $\operatorname{VDE}(\infty)-\mathrm{VDE}_{\max }$, and the associated times $t_{\min }, t_{\max }$, and $\tau(\infty)$. $\mathrm{VDE}_{\text {min }}$ ranges between $100-180 \mathrm{meV}$ and does not show any systematic size dependence. In contrast, $\mathrm{VDE}_{\max }$ and $\operatorname{VDE}(\infty)$ values cover a wider range and grow monotonically from $n=$ 5 to 10 . Initial detachment energies $\operatorname{VDE}(0)$ also spread over a broad range and show a systematic increase, although a discontinuity can be observed between $n=8$ and 9 , coincident with the decrease of the pump wavelength.

\section{Discussion}

5.1. Energetics. Time-resolved photoelectron spectra are dominated by the $\left[1+1^{\prime}\right]$ excited-state detachment signal and an intense feature from slow electrons emitted near eKE $\approx 0$ $\mathrm{eV}$. In agreement with the VDE values of $\mathrm{I}^{-}\left(\mathrm{CH}_{3} \mathrm{CN}\right)_{n}$ clusters measured by Markovich et al., ${ }^{26}$ no direct photodetachment was observed, as these binding energies exceeded the pump photon energies in our experiments. The CTTS transition in clusters with $n \leq 8$ was induced by $4.68 \mathrm{eV}$ pump photons. Larger clusters showed only weak excited-state signals at this pump photon energy and thus were pumped at $5.06 \mathrm{eV}$. The blue shift 
TABLE 1: Summarized Energetics and Time Constants for the Relaxation of the CTTS State in $\mathrm{I}^{-}\left(\mathrm{CH}_{3} \mathrm{CN}\right)_{n}$ after Excitation ${ }^{a}$

\begin{tabular}{lcccccccccc}
\hline$n$ & $\mathrm{VDE}(0)(\mathrm{eV})$ & $\mathrm{VDE}_{\min }(\mathrm{eV})$ & $\mathrm{VDE}_{\max }(\mathrm{eV})$ & $\mathrm{VDE}(\infty)(\mathrm{eV})$ & $\Delta E_{1}(\mathrm{meV})$ & $\Delta E_{2}(\mathrm{meV})$ & $\Delta E_{3}(\mathrm{meV})$ & $t_{\min }(\mathrm{fs})$ & $t_{\max }(\mathrm{ps})$ & $\tau_{\infty}(\mathrm{ps})$ \\
\hline 5 & 0.302 & 0.176 & 0.480 & 0.421 & -126 & 304 & -59 & 331 & 12.4 & 109 \\
6 & 0.364 & 0.139 & 0.502 & 0.456 & -225 & 363 & -46 & 376 & 13.0 & 146 \\
7 & 0.411 & 0.149 & 0.537 & 0.506 & -262 & 388 & -31 & 386 & 15.9 & $(58)$ \\
8 & 0.448 & 0.127 & 0.538 & 0.507 & -321 & 411 & -31 & 401 & 17.8 \\
9 & 0.357 & 0.100 & 0.548 & & -257 & 448 & & 425 & \\
10 & 0.426 & 0.165 & 0.578 & & -261 & 413 & & 502
\end{tabular}

${ }^{a}$ Excitation at $h v_{\text {pump }}=4.68 \mathrm{eV}(n=5-8)$ and $5.06 \mathrm{eV}(n=9,10)$. Statistical uncertainties of absolute detachment energies amount to 60 $\mathrm{meV}$, of relative energies $25 \mathrm{meV}, 50$ fs for $t_{\min }, 3.0 \mathrm{ps}$ for $t_{\max }$, and $50 \mathrm{ps}$ for $\tau_{\infty}$.

of the charge transfer band is in agreement with the detachment energies increasing from 4.95 to $5.71 \mathrm{eV}$ in the cluster size range studied here ${ }^{26}$ and the expected maximum of the CTTS band being located slightly below the continuum. ${ }^{44}$ The pump photon energy only allows for charge transfer to the manifold corresponding to the ${ }^{2} \mathrm{P}_{3 / 2}$ state of the neutral iodine atom, whereas the band converging to the spin-orbit excited state $\left(\Delta E_{\mathrm{SO}}=\right.$ $0.94 \mathrm{eV}$ ) is not accessible.

The overall change in VDE of the excited-state signal is strongly reminiscent of the modulations observed after excitation of the CTTS band in $\mathrm{I}^{-}\left(\mathrm{H}_{2} \mathrm{O}\right)_{n}$. These have been attributed to electron hydration dynamics within the cluster, most likely leading to a surface-bound electron in the size-range studied thus far. ${ }^{19}$ Bare acetonitrile cluster anions, however, do not exhibit such a conformation. The $\operatorname{VDE}(\infty)$ values found here resemble those of isomer I of $\left(\mathrm{CH}_{3} \mathrm{CN}\right)_{n}{ }^{-}$clusters in which the electron resides in a solvent cavity. ${ }^{22,23}$ There is no evidence for the formation of more strongly bound isomer II, assigned to a valence-bound electron, even at higher probe photon energies ( $h v_{\text {probe }}=3.12 \mathrm{eV}$, not shown). With these considerations in mind, we now consider the question of how the charge transfer and solvation dynamics proceed in detail.

5.2. CTTS Dynamics. In this section, the time-evolution of the photoelectron spectra is considered, and the underlying dynamics responsible for this evolution are discussed. Taking Figures 3 and 6 as references, we would like to understand the initial $\operatorname{VDE}$ value, $\operatorname{VDE}(0)$, the abrupt drop to $\mathrm{VDE}_{\text {min }}$ after $330 \mathrm{fs}$, the biexponential increase toward $\mathrm{VDE}_{\max }$ with time constants $\tau_{1}$ and $\tau_{2}$, and, for the smaller $(n=5-8)$ clusters, the slight drop in VDE over the 100 ps time scale. The $\sim 330$ fs delay between the initial increase in photoelectron yield and the drop in VDE is also of interest. We consider the earliest and latest times first, since these are the most straightforward to interpret, and then focus on the intermediate dynamics.

The initial step in our experiments is the pump-induced excitation of the CTTS band,

$$
\mathrm{I}^{-}\left(\mathrm{CH}_{3} \mathrm{CN}\right)_{n} \stackrel{\mathrm{h} v}{\rightarrow}\left[\mathrm{I}^{\bullet}\left(\mathrm{CH}_{3} \mathrm{CN}\right)_{n}^{-}\right]^{*}
$$

transferring the originally localized excess charge from the interior halide into a diffuse charge transfer state characterized by $\operatorname{VDE}(0)$ values ranging from $302-448 \mathrm{meV}$. These values are consistent with resonant $\left[1+1^{\prime}\right]$ detachment and $\mathrm{VDE}_{\mathrm{dir}}$ values determined independently by Markovich et al. ${ }^{26}$ by onephoton direct detachment. In other words, the VDE of the initially excited-state is given by $\operatorname{VDE}(0)=\mathrm{VDE}_{\mathrm{dir}}-h v_{\text {pump }}$. In Figure 6, the increase of $\operatorname{VDE}(0)$ from $n=5-8$ is a consequence of the monotonic increase of $\mathrm{VDE}_{\mathrm{dir}}$ with $n$, whereas the step between $n=8$ and 9 results from the higher pump photon energy used for the $n=9$ and 10 clusters.

We next consider the long-time dynamics. The presence of the very low-energy electrons indicates that the excited CTTS state decays by autodetachment. A similar effect was seen in $\mathrm{I}^{-}\left(\mathrm{H}_{2} \mathrm{O}\right)_{n}$ clusters. ${ }^{19}$ In those experiments, the autodetachment signal was depleted just after the probe pulse but then recovered on the same time-scale upon which the excited-state signal decayed, yielding the lifetime of the CTTS state with respect to autodetachment. These lifetimes varied from $8 \mathrm{ps}(n=4)$ to 3 ns $(n=10)$. In the $\mathrm{I}^{-}\left(\mathrm{CH}_{3} \mathrm{CN}\right)_{n}$ experiments, probe-induced depletion of the autodetachment signal is observed, but there is neither recovery of this signal nor decay of the pump-probe signal by $200 \mathrm{ps}$, the longest delay time used here. Hence, while the initially produced CTTS state evolves into a state that eventually decays by autodetachment, the lifetime of this state is at least 200 ps.

The nature of this long-time state is suggested by comparison to the PE spectra by Mitsui et al. ${ }^{22}$ on $\left(\mathrm{CH}_{3} \mathrm{CN}\right)_{n}{ }^{-}$clusters. Our $\mathrm{VDE}_{\max }$ values are in reasonable agreement with their VDEs for bare acetonitrile cluster anions in the isomer I conformation, that is, clusters in which the electron resides in a solvent cavity. Mitsui et al. observed their onset of formation for $n=10$ and report VDEs of 430-530 $\mathrm{meV}$ in the size range $n=10-12$. Linear extrapolation of these values to the size range observed in our experiments leads to very similar values within $\sim 0.1 \mathrm{eV}$ of our observed values of $\mathrm{VDE}_{\max }$. These small differences, which are significantly smaller than the spectral widths, imply that the states seen at long times in our experiments are $\left(\mathrm{CH}_{3} \mathrm{CN}\right)_{n}{ }^{-}$clusters with internally solvated electrons.

It thus appears that the overall dynamics in the time-resolved PE experiments track the evolution of the initial $\mathrm{I}^{-}\left(\mathrm{CH}_{3} \mathrm{CN}\right)_{n}$ state, in which the iodide is internally solvated, ${ }^{26-29}$ to a bare acetonitrile cluster anion with an internally solvated electron. The direct molecular dynamics calculations by Takayanagi on $\mathrm{I}^{-}\left(\mathrm{CH}_{3} \mathrm{CN}\right)_{3}$ provide useful insights into this mechanism. In the initial geometry, the $\mathrm{I}^{-}$lies at the center of a 3-fold symmetric solvent cage; each acetonitrile is oriented with its $\mathrm{CH}_{3}$ group pointing toward the iodide, as expected because of the highly electronegative $\mathrm{CN}$ group. A similar structure is found for $\left(\mathrm{CH}_{3} \mathrm{CN}\right)_{3}{ }^{-}$, except that the cavity radius is slightly smaller, $3.55 \AA$ as opposed to $3.85 \AA$ in $\mathrm{I}^{-}\left(\mathrm{CH}_{3} \mathrm{CN}\right)_{3}$. Hence, the diffuse electron resulting from CTTS excitation is formed in a rather confined space, and significant repulsive interactions with both the I atom and solvent cage are expected. This is confirmed by the simulations, which show the solvent cage enlarging, the excess electron localizing on one or two $\mathrm{CH}_{3} \mathrm{CN}$ molecules, and the I atom moving away from the solvent cavity, all within the first $800 \mathrm{fs}$ after CTTS excitation.

Our experimental results are consistent with several aspects of this calculation. The initial drop in $\operatorname{VDE}$, from $\operatorname{VDE}(0)$ to $\mathrm{VDE}_{\min }$, over the first $300 \mathrm{fs}$, can indeed be explained by rapid expansion of the cavity accompanied by localization of the electron on a smaller number of solvent molecules. Localization, in particular, can result in a significantly reduced VDE, given that a single acetonitrile molecule can only bind an excess electron in a very loosely bound dipole-bound state, with a 
binding energy of only a few millielectronvolts. ${ }^{49}$ The rapid drop in integrated excited-state intensity for $\mathrm{I}^{-}\left(\mathrm{CH}_{3} \mathrm{CN}\right)_{5}$ over the first 300 fs (see Figure 3) may also reflect these dynamics, because the rapidly changing nature of the excess electron wave function is likely to affect its photodetachment cross-section. The biexponential increase in VDE over the next 1-2 ps, from $\mathrm{VDE}_{\text {min }}$ to $\mathrm{VDE}_{\max }$, described by eq 3 , could result from a combination of ejection of the I atom from the solvent cavity and reorganization of the solvent molecules to accommodate the excess electron inside a smaller cavity. This reorganization can involve reorientation of the solvent molecules as well as their pulling together around the electron; according to the molecular dynamics simulations by Markovich et al., ${ }^{26}$ the six solvent molecules in $\mathrm{I}^{-}\left(\mathrm{CH}_{3} \mathrm{CN}\right)_{6}$ form a ring around the central iodide in a "head-to-tail" configuration, with the methyl group of each solvent molecule in close proximity to the $\mathrm{CN}$ group of the adjacent molecule, while calculations on isomer I of $\left(\mathrm{CH}_{3} \mathrm{CN}\right)_{6}{ }^{-}$find the electron in an octahedral cavity with all $\mathrm{CN}$ groups pointing outward. ${ }^{23}$

Finally, we consider the $\sim 100$ ps decrease in VDE of $59-31$ meV, seen for the $n=5-8$ clusters. This shift is most likely from evaporation of either a solvent molecule or I atom from the cluster. The magnitude of this shift is similar to the stabilization energy in bare isomer I clusters of $\Delta E \approx 50 \mathrm{meV}$ upon addition of a single solvent molecule in the size range $n$ $=10-12$, as observed by Mitsui et al. ${ }^{22}$ However, it is also comparable to a similar shift in $\mathrm{I}^{-}\left(\mathrm{H}_{2} \mathrm{O}\right)_{n}$ clusters that was attributed to loss of a weakly bound I atom, ${ }^{19}$ an interpretation that was supported by subsequent calculations by Takayanagi. ${ }^{50}$ Note that loss of an I atom from the cluster may be distinct from ejection of the I atom from the solvent cavity, as it may get trapped in a shallow attractive well outside the solvent network and thus leave the cluster at a much longer time. In any case, it is difficult to distinguish between these two possible channels based on energetic shifts alone.

5.3. Comparison to $\mathrm{I}^{-}\left(\mathrm{H}_{2} \mathrm{O}\right)_{n}$. A primary motivation of this study was to compare it to similar work on $\mathrm{I}^{-}\left(\mathrm{H}_{2} \mathrm{O}\right)_{n}$ clusters. ${ }^{17-19}$ Pump/probe PE spectroscopy of $\mathrm{I}^{-}\left(\mathrm{H}_{2} \mathrm{O}\right)_{n}$ with $n \leq 10$ showed a transient state undergoing qualitatively similar modulations of VDEs as in our current work. There is an initial drop in the VDE just after excitation, followed by an increase, $\Delta E_{2}$ over $\tau_{2}=0.2-1.4 \mathrm{ps}$. In a final relaxation step, $\left(\tau_{3}=25-90 \mathrm{ps}\right)$ electron binding energies decreased again, before the transient decayed by delayed emission of slow electrons $\left(\tau_{\mathrm{CTTS}}=8 \mathrm{ps}\right.$ to 3 ns). Despite the similarities, a closer look reveals fundamental differences in the observed dynamics and energetics. The initial drop in VDE was much faster in $\mathrm{I}^{-}\left(\mathrm{H}_{2} \mathrm{O}\right)_{n}$ clusters, essentially limited by the cross-correlation of the two laser pulses. Both $\tau_{2}$ and $\Delta E_{2}$ increased with $n$ for $\mathrm{I}^{-}\left(\mathrm{H}_{2} \mathrm{O}\right)_{n}$ clusters, while the analogous measurements in $\mathrm{I}^{-}\left(\mathrm{CH}_{3} \mathrm{CN}\right)_{n}$ show a much weaker size-dependence. Moreover, the increase in VDE could be described by a single exponential for $\mathrm{I}^{-}\left(\mathrm{H}_{2} \mathrm{O}\right)_{n}$, as opposed to the biexponential dynamics seen here. Finally, as discussed above, even though the states created by CTTS excitation for both cluster types decay by autodetachment, the time scale for this decay was outside our observation window of 200 ps for $\mathrm{I}^{-}\left(\mathrm{CH}_{3} \mathrm{CN}\right)_{n}$ clusters.

These differences can be attributed in part to the different initial and final conditions in the two sets of experiments. As discussed in Section 1, for $n \leq 10$, both the iodide anion in $\mathrm{I}^{-}\left(\mathrm{H}_{2} \mathrm{O}\right)_{n}$ and excess electron in $\left(\mathrm{H}_{2} \mathrm{O}\right)_{n}{ }^{-}$are bound to the cluster surface rather than located inside a solvent cavity. As a result, the repulsive interactions just after CTTS excitation should be mitigated in $\mathrm{I}^{-}\left(\mathrm{H}_{2} \mathrm{O}\right)_{n}$ clusters, because the electron is not being produced in such a confined volume. This may explain the differences seen at the earliest times. Moreover, in $\mathrm{I}^{-}\left(\mathrm{H}_{2} \mathrm{O}\right)_{n}$ clusters, the solvent reorganization leading to stabilization of the excess electron does not require the I atom to leave a solvent cavity, possibly accounting for the monoexponential vs biexponential increase in the VDE. The apparently shorter lifetime of the excited-state in $\mathrm{I}^{-}\left(\mathrm{H}_{2} \mathrm{O}\right)_{n}$ may reflect the more facile escape of a surface-bound electron as opposed to an interior electron. Overall, comparison of the two cluster types enables one to probe how solvation dynamics differ at the surface versus interior of small clusters.

\section{Summary}

We have used time-resolved photoelectron spectroscopy to study photoinduced charge transfer in mass-selected, iodidedoped acetonitrile clusters $\mathrm{I}^{-}\left(\mathrm{CH}_{3} \mathrm{CN}\right)_{n}$ with $n=5-10$ after excitation at $4.68 \mathrm{eV}(n=5-8)$ and $5.06 \mathrm{eV}(n=9,10)$. The photoelectron spectra yield time-varying VDEs, which drop rapidly within $300-400$ fs of the excitation pulse, then recover biexponentially to reach their maximum values with time constants of $250 \pm 100 \mathrm{fs}$ and $2.5 \pm 1.0 \mathrm{ps}$. The $n=5-8$ clusters then show a drop in VDE of around $50 \mathrm{meV}$ on a much longer time scale. Although the excited-state created by the pump pulse decays by emission of a slow electron, this decay occurs beyond our experimental time window of 200 ps. Comparison to theory suggests that the initial drop in VDE comes from the response of the cluster to the pump-induced formation of a diffuse electron cloud within the solvent cavity originally containing the iodide anion. The cavity expands, and the electron localizes on one or two acetonitrile molecules, resulting in a transition state with a low VDE. The subsequent rise in VDE is attributed to the I atom leaving the cavity and rearrangement of the solvent molecules to stabilize the excess electron, ultimately forming an acetonitrile cluster anion with an internally solvated electron. The $50 \mathrm{meV}$, long-time decrease in VDE for $n=5-8$ is most likely from evaporation of an I atom or solvent molecule.

Charge-transfer-to-solvent dynamics in the small clusters studied here are quite distinct from the analogous condensed phase process, even though the larger clusters in our experiment approach closure of the first solvation shell. ${ }^{26}$ Obviously, cluster sizes in the current work are too small to allow for disorder in the solvent network, a requirement to observe direct emission of the excess charge in preformed solvent cavities or direct formation of valence anions by electron attachment to a prealigned antiparallel acetonitrile dimer, as seen in the condensed phase. ${ }^{20,21}$ Therefore, our experiments may resemble most closely the initial dynamics in the creation of the $\left(\mathrm{I}: \mathrm{e}^{-}\right)$ contact pair. ${ }^{20}$ Unfortunately, condensed phase measurements have so far not focused on this very early stage in the formation of solvated electrons, which should be also completed within the first few picoseconds after CTTS excitation. Research on early dynamics in the bulk, as well as gas phase spectroscopy on larger clusters, will be necessary to bridge the gap and allow for a seamless description of the charge transfer process.

Acknowledgment. This research is supported by the National Science Foundation under Grant No. CHE-0649647. OTE is grateful to the A. von Humboldt Foundation (Germany) for the award of a Feodor-Lynen fellowship.

\section{References and Notes}

(1) Clark, C.; Zika, R. Marine Organic Photochemistry: From the Sea Surface to Marine Aerosols. In Marine Chemistry; Springer: Berlin, 2000; pp $1-33$. 
(2) Jungwirth, P.; Tobias, D. J. J. Phys. Chem. B 2002, 106, 6361.

(3) Boudaiffa, B.; Cloutier, P.; Hunting, D.; Huels, M. A.; Sanche, L. Science 2000, 287, 1658.

(4) Coe, J. V. Int. Rev. Phys. Chem. 2001, 20, 33.

(5) Chen, X. Y.; Bradforth, S. E. Annu. Rev. Phys. Chem. 2008, 59, 203.

(6) Walhout, P. K.; Alfano, J. C.; Kimura, Y.; Silva, C.; Reid, P. J.; Barbara, P. F. Chem. Phys. Lett. 1995, 232, 135.

(7) Shi, X. L.; Long, F. H.; Eisenthal, K. B. J. Phys. Chem. 1995, 99, 6917.

(8) Chandrasekhar, N.; Krebs, P.; Unterreiner, A. N. J. Chem. Phys. 2006, 125,7 .

(9) Barthel, E. R.; Martini, I. B.; Keszei, E.; Schwartz, B. J. J. Chem. Phys. 2003, 118, 5916.

(10) Bragg, A. E.; Schwartz, B. J. J. Phys. Chem. B 2008, 112, 483.

(11) Bragg, A. E.; Schwartz, B. J. J. Phys. Chem. A 2008, 112, 3530.

(12) Ferro, Y.; Allouche, A.; Kempter, V. J. Chem. Phys. 2004, 120, 8683.

(13) Shkrob, I. A. J. Phys. Chem. A 2006, 110, 3967.

(14) Lindner, J.; Unterreiner, A. N.; Vöhringer, P. ChemPhysChem 2006, 7, 363 .

(15) Long, F. H.; Shi, X. L.; Lu, H.; Eisenthal, K. B. J. Phys. Chem. 1994, 98, 7252 .

(16) Kloepfer, J. A.; Vilchiz, V. H.; Lenchenkov, V. A.; Germaine, A. C.; Bradforth, S. E. J. Chem. Phys. 2000, 113, 6288.

(17) Lehr, L.; Zanni, M. T.; Frischkorn, C.; Weinkauf, R.; Neumark, D. M. Science 1999, 284, 635.

(18) Verlet, J. R. R.; Kammrath, A.; Griffin, G. B.; Neumark, D. M. J. Chem. Phys. 2005, 123, 231102.

(19) Kammrath, A.; Verlet, J. R. R.; Bragg, A. E.; Griffin, G. B. Neumark, D. M. J. Phys. Chem. A 2005, 109, 11475.

(20) Xia, C. G.; Peon, J.; Kohler, B. J. Chem. Phys. 2002, 117, 8855

(21) Shkrob, I. A.; Sauer, M. C. J. Phys. Chem. A 2002, 106, 9120.

(22) Mitsui, M.; o, N.; Kokubo, S.; Nakajima, A.; Kaya, K. Phys. Rev. Lett. 2003, 91, 153002 679 .

(23) Takayanagi, T.; Hoshino, T.; Takahashi, K. Chem. Phys. 2006, 324,

(24) Timerghazin, Q. K.; Peslherbe, G. H. J. Phys. Chem. B 2008, 112, 520 .

(25) Dessent, C. E. H.; Bailey, C. G.; Johnson, M. A. J. Chem. Phys 1995, 103, 2006.

(26) Markovich, G.; Perera, L.; Berkowitz, M. L.; Cheshnovsky, O. J. Chem. Phys. 1996, 105, 2675.
(27) Nguyen, T. N. V.; Peslherbe, G. H. J. Phys. Chem. A 2003, 107, 1540.

(28) Nguyen, T. N. V.; Hughes, S. R.; Peslherbe, G. H. J. Phys. Chem. $B$ 2008, $112,621$.

(29) Takayanagi, T. J. Phys. Chem. A 2006, 110, 7011.

(30) Koch, D. M.; Peslherbe, G. H. Chem. Phys. Lett. 2002, 359, 381.

31) Robertson, W. H.; Johnson, M. A. Annu. Rev. Phys. Chem. 2003, 54,173

(32) Neumark, D. M. Mol. Phys. 2008, 106, 2183

(33) Bragg, A. E.; Verlet, J. R. R.; Kammrath, A.; Cheshnovsky, O.; Neumark, D. M. J. Am. Chem. Soc. 2005, 127, 15283.

(34) Verlet, J. R. R.; Bragg, A. E.; Kammrath, A.; Cheshnovsky, O.; Neumark, D. M. Science 2005, 307, 93.

(35) Kammrath, A.; Verlet, J. R. R.; Griffin, G. B.; Neumark, D. M. J. Chem. Phys. 2006, 125, 076101.

(36) Barnett, R. N.; Landman, U.; Cleveland, C. L.; Jortner, J. J. Chem. Phys. 1988, 88, 4429.

(37) Coe, J. V.; Lee, G. H.; Eaton, J. G.; Arnold, S. T.; Sarkas, H. W.; Bowen, K. H.; Ludewigt, C.; Haberland, H.; Worsnop, D. R. J. Chem. Phys. 1990, 92, 3980.

(38) Turi, L.; Sheu, W. S.; Rossky, P. J. Science 2005, 309, 914.

(39) Shin, J. W.; Hammer, N. I.; Headrick, J. M.; Johnson, M. A. Chem. Phys. Lett. 2004, 399, 349.

(40) Roscioli, J. R.; Hammer, N. I.; Johnson, M. A. J. Phys. Chem. A 2006, 110,7517 .

(41) Sommerfeld, T.; Jordan, K. D. J. Am. Chem. Soc. 2006, 128, 5828.

(42) Asmis, K. R.; Santambrogio, G.; Zhou, J.; Garand, E.; Headrick, J.; Goebbert, D.; Johnson, M. A.; Neumark, D. M. J. Chem. Phys. 2007, 126,5 .

(43) Rabinowitch, E. Rev. Mod. Phys. 1942, 14, 112.

(44) Serxner, D.; Dessent, C. E. H.; Johnson, M. A. J. Chem. Phys. 1996, 105,7231 . 31.

(45) Timerghazin, Q. K.; Peslherbe, G. H. Chem. Phys. Lett. 2002, 354,

(46) Davis, A. V.; Wester, R.; Bragg, A. E.; Neumark, D. M. J. Chem. Phys. 2003, 118, 999.

(47) Griffin, G. B.; Kammrath, A.; Ehrler, O. T.; Young, R. M.; Cheshnovsky, O.; Neumark, D. M. Chem. Phys. 2008, 350, 69.

(48) Dribinski, V.; Ossadtchi, A.; Mandelshtam, V. A.; Reisler, H. Rev. Sci. Instrum. 2002, 73, 2634.

(49) Bailey, C. G.; Dessent, C. E. H.; Johnson, M. A.; Bowen, K. H. J. Chem. Phys. 1996, 104, 6976.

(50) Takahashi, K.; Takayanagi, T. Chem. Phys. 2007, 342, 95. 